\title{
Remarkable Response to Cisplatin Doublet Chemotherapy in Pulmonary Metastasis With Left Atrial Extension From a Nasopharyngeal Cancer
}

\author{
Frank S. Fan ${ }^{1}$, Chen-Feng Chiu ${ }^{2}$, Hsuan-hua Huang ${ }^{3}$, Hwei-Fan Wendy Shu ${ }^{3}$ \\ 1. Section of Hematology and Oncology, Ministry of Health and Welfare Taitung Hospital, Taitung County, TWN 2. \\ Section of Pulmonology, Department of Internal Medicine, Ministry of Health and Welfare Feng Yuan Hospital, \\ Taichung City, TWN 3. Pathology, Ministry of Health and Welfare Feng Yuan Hospital, Taichung City, TWN
}

Corresponding author: Frank S. Fan, fantast.fan@msa.hinet.net

\begin{abstract}
A 60 -year-old male patient whose nasopharyngeal carcinoma was brought to complete remission with induction chemotherapy composing of cisplatin plus fluorouracil and subsequent radiotherapy with intent of cure eight years ago presented with dyspnea due to left side massive pleural effusion with pleural seedings, left lower lobe huge space occupying lesion, and left atrial tumor extending from the intrapulmonary lesion through left inferior pulmonary veins. Pleural biopsy revealed a picture of nonkeratinizing squamous cell carcinoma positive for Epstein-Barr virus-encoded small RNAs in situ hybridization, leading to a diagnosis of late pulmonary metastases from the antecedent nasopharyngeal carcinoma. Systemic chemotherapy with initial cisplatin plus paclitaxel and subsequent cisplatin plus gemcitabine brought remarkable resolution to the malignant cardiac and intrathoracic lesions. So far as we know, this is the first case report of left atrial invasion from pulmonary metastasis of a nasopharyngeal carcinoma origin in the English literature.
\end{abstract}

Categories: Cardiac/Thoracic/Vascular Surgery, Oncology, Pulmonology

Keywords: nasopharyngeal cancer, pulmonary metastasis, left atrium invasion, epstein-barr virus, chemotherapy, paclitaxel, cisplatin, gemcitabine

\section{Introduction}

Tumor invasion into pulmonary veins or left atrium from lung cancer is a not-so-rare phenomenon. The incidence was found to be $9.3 \%$ as evaluated with CT scan [1]. Despite multidisciplinary therapeutic approach, mainly surgical resection, the prognosis of such advanced disease status was poor with a five-year survival of $14 \%$ only [2]. Interestingly, left atrial extension from pulmonary metastasis of other primary sites has also been reported in a variety of occasional cases including Ewing sarcoma, renal cell carcinoma, colon cancer, rectal cancer, chondrosarcoma, and cervix cancer [3-8]. Herein, we present an extraordinary case of nasopharyngeal carcinoma which, initially brought to complete response by induction chemotherapy and local radiotherapy, recurred eight years later as a pulmonary metastasis with invasion into left atrium through left pulmonary veins. To the best of our knowledge, this is the first report of left atrial involvement

Review began 02/22/2021 Review ended 03/03/2021 Published 03/09/2021

\section{(c) Copyright 2021}

Fan et al. This is an open access article distributed under the terms of the Creative Commons Attribution License CC-BY 4.0., which permits unrestricted use, distribution, and reproduction in any medium, provided the original author and source are credited. by nasopharyngeal carcinoma in the English literature.

\section{Case Presentation}

A 60-year-old single male patient went to a regional teaching hospital in central Taiwan for help in May 2020. His chief complaints were progressive exertional dyspnea and shortness of breath for one month. Poor appetite and body weight loss were also noted. He had a cigarette smoking history of more than 30-packageyear. Laboratory examination revealed normal white cell count but mild microcytic anemia with hemoglobin $10 \mathrm{~g} / \mathrm{dL}$, mean corpuscular volume $73.3 \mathrm{fl}$, probably due to an alpha-thalassemia trait, and moderate thrombocytosis with platelet count $630,000 / \mathrm{mL}$, most likely reactive to the underlying malignancy. There was no iron deficiency. His blood sugar, liver, and renal functions were within normal reference ranges. Serum tumor markers including carcinoembryogenic antigen, cancer antigen 19.9, and squamous cell carcinoma antigen were not elevated.

Chest X-ray examination revealed massive left side pleural effusion pushing heart shadow rightward (Figure 1A). For relieving respiratory distress, thoracocentesis with insertion of a draining catheter into the left pleural cavity (Figure $1 B$ ) and a left pleural biopsy were performed along with pleural effusion cytology studies. CT scan showed a huge mass, $9.3 \mathrm{~cm}$ in diameter, in the left lower lobe of the lung with tumor extension into left atrium through left inferior pulmonary veins (Figure 2). There were also obvious thickened pleural seedings. 


\section{Cureus}

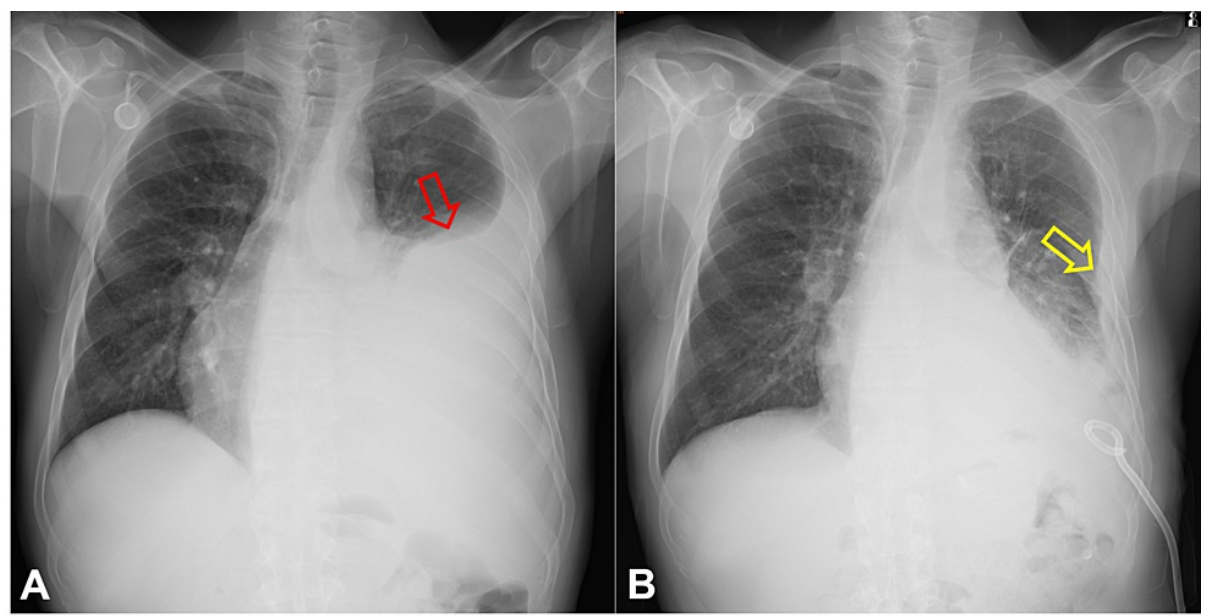

\section{FIGURE 1: Chest X-ray films of the patient at presentation.}

A. Left side massive pleural effusion (red arrow) (May 6, 2020). B. Pleural thickening clearly seen (yellow arrow) after pleural effusion drainage with a pigtail catheter (May 18, 2020).

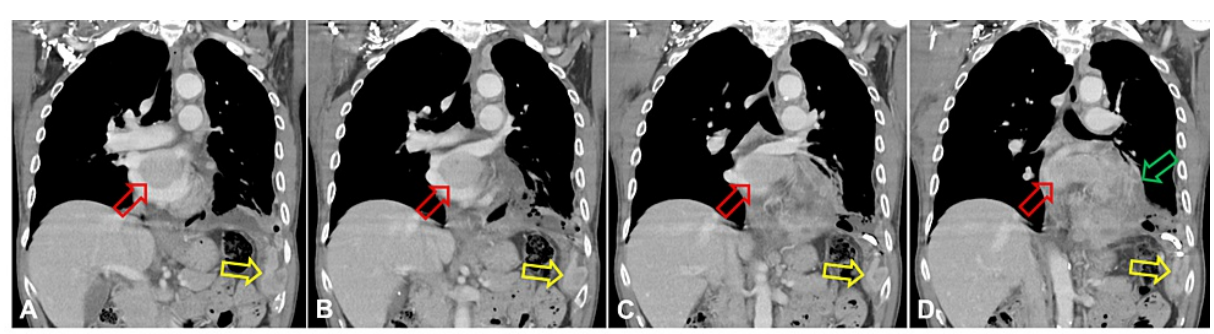

FIGURE 2: CT scan of chest at initial presentation (May 11, 2020).

A-D: Subsequent coronal views. Red arrow: Tumor invasion into the left atrium from left inferior pulmonary veins. Yellow arrow: Pleural metastasis. Green arrow: Metastatic tumor mass occupying left lower lobe and pleura.

The biopsy result turned out to be a nonkeratinizing squamous cell carcinoma (Figure 3). Since the patient had a history of nasopharyngeal carcinoma which was brought to complete remission after induction chemotherapy with one cycle of cisplatin plus continuous fluorouracil and subsequent radiotherapy at a tertiary hospital in 2012, in situ hybridization for Epstein-Barr virus-encoded small RNAs (EBER) was ordered for the present pleural biopsy and the first-hand nasopharyngeal pathologic specimen retrieved from the previous hospital. A CT scan of neck did not detect evidence of recurrence in nasopharynx or any regional lymph node metastasis.
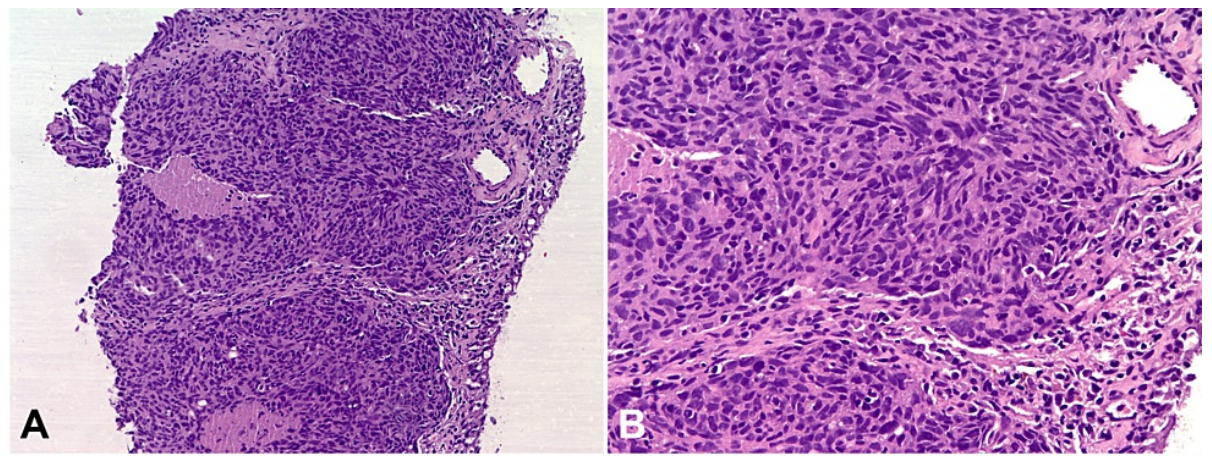

\section{FIGURE 3: Pathology study of the pleural biopsy.}

Metastatic nonkeratinizing squamous cell carcinoma (hematoxylin and eosin stain; A. x100. B. x200). 


\section{Cureus}

Because EBER study of both the present pleural and the original nasopharyngeal biopsies gave positive results and the microscopic pictures disclosed somewhat similarity in morphological characteristics (Figure 4), a diagnosis of pulmonary metastasis from an antecedent nasopharyngeal carcinoma is thus established. The squamous cell carcinoma marker $\mathrm{p} 40$ was also positive in samples taken from the earlier nasopharynx and the present pleura, respectively, with a time interval of eight years in between.

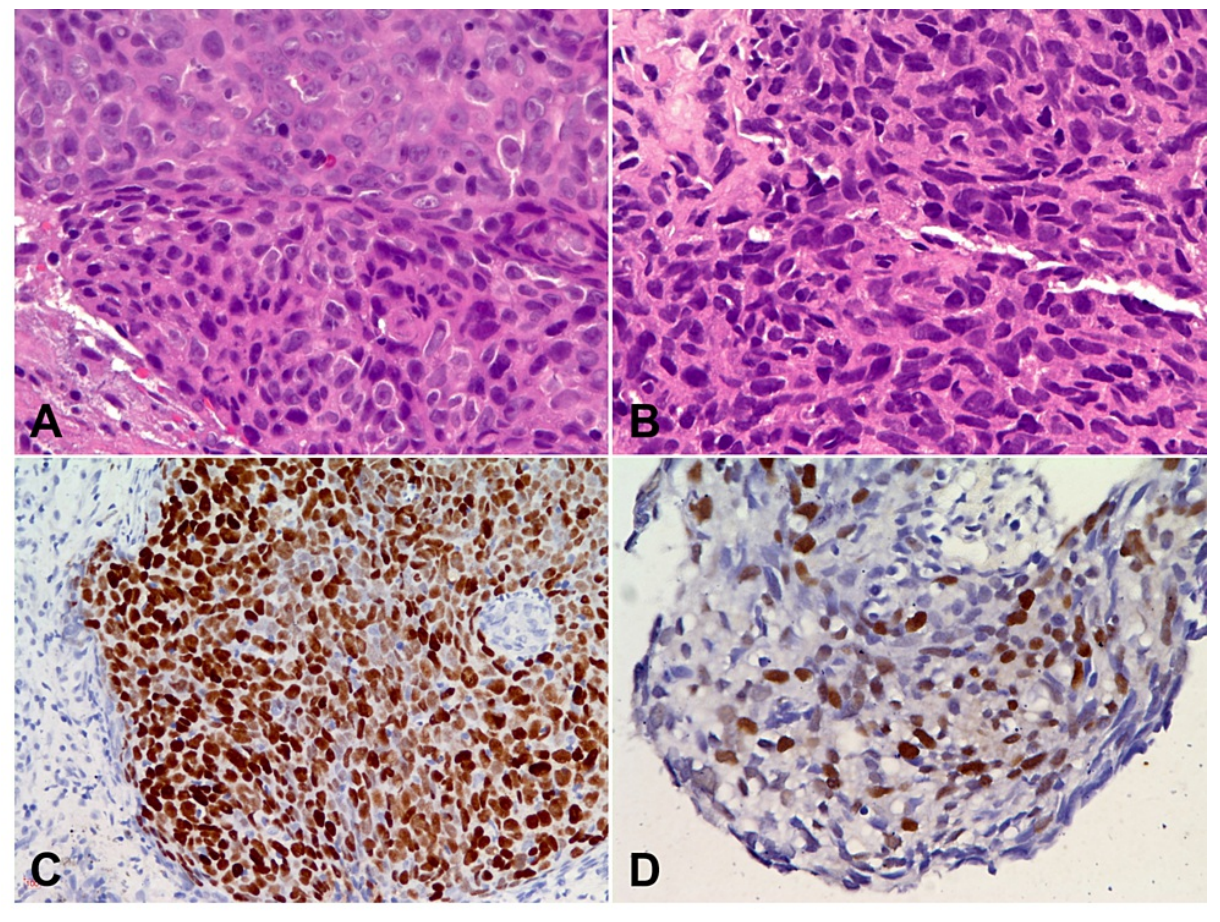

\section{FIGURE 4: Pathology study of the primary and the late metastatic lesions.}

$A$ and $C$. The original nasopharyngeal carcinoma. B and D. The present pleural metastasis. A and B: Hematoxylin and eosin stain (x400). C and D: EBER in situ hybridization (x400).

EBER, Epstein-Barr virus-encoded small RNA

The patient took our advice and agreed for a systemic combination chemotherapy with cisplatin $(100 \mathrm{mg} / \mathrm{m} 2)$ and paclitaxel $(175 \mathrm{mg} / \mathrm{m} 2)$. Surprisingly, the pulmonary lesions responded dramatically to the first course of chemotherapy (Figure 5). He was subsequently scheduled to take the chemotherapy every four weeks as the first step of therapy. Although the treatment plan was interrupted for a short period of time due to left side pneumonia with impending septic shock after the second course (Figure 6A), he eventually recovered (Figure 6B) and completed totally four courses of cisplatin/paclitaxel chemotherapy from June to September 2020. Although follow-up study with CT scan revealed a very good response with dramatic shrinkage of tumors in the pleura, lung, and heart, complete resolution of the lesions was not achieved (Figure 7A-F). He then received four more courses of chemotherapy composing of cisplatin $(100 \mathrm{mg} / \mathrm{m} 2)$ on day 1 plus gemcitabine $(1000 \mathrm{mg} / \mathrm{m} 2)$ on day 1 and 8 from October 2020 to January 2021. Interestingly, this second step of therapy brought nearly complete remission of the metastatic pulmonary lesions and left atrial tumor invasion (Figure $7 G, \mathrm{H}$ ). 


\section{Cureus}

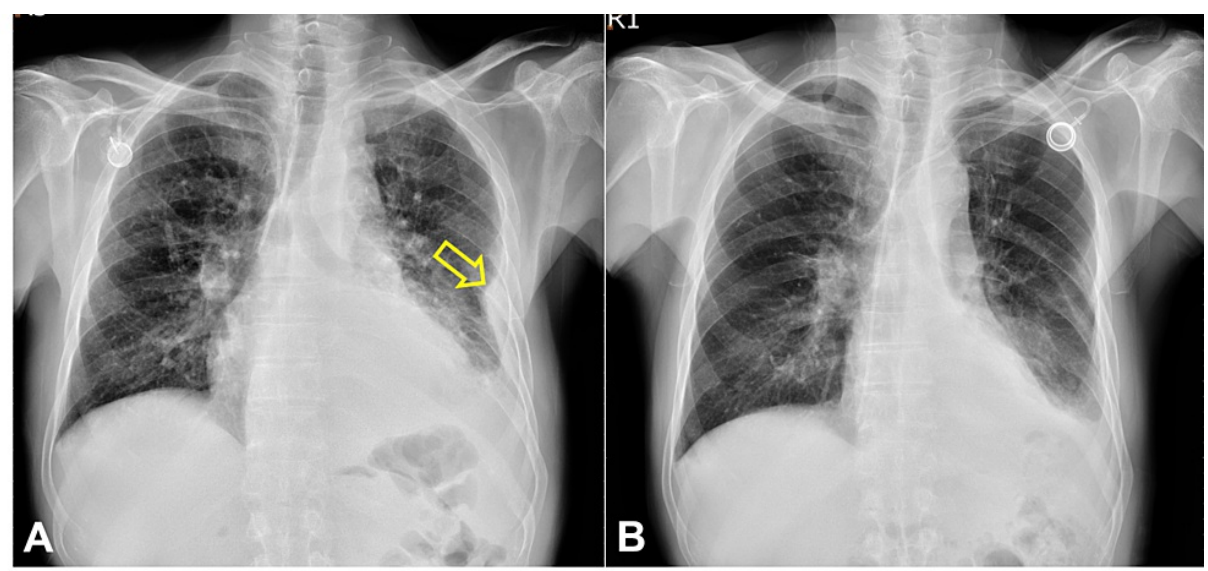

FIGURE 5: Chest X-ray films of the patient at the initial stage of treatment .

A. After removal of the pigtail drainage catheter five days prior to the first course of cisplatin/paclitaxel chemotherapy (Jun. 8, 2020). Yellow arrow: pleural tumor seeding. B. Reimplantation of a Port-A catheter due to malfunction of the old one 25 days after the first course of cisplatin/paclitaxel chemotherapy (Jul. 8, 2020). Very good response in pleural seeding and effusion could be seen.

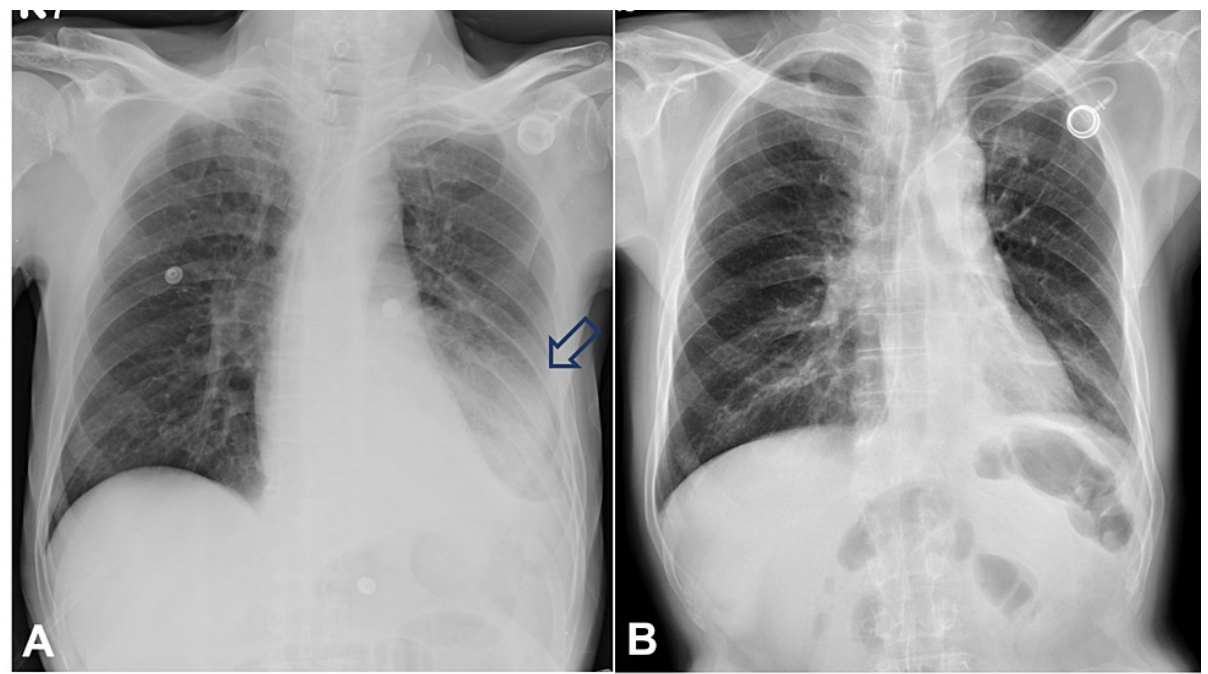

FIGURE 6: Chest X-ray films of the patient during treatment.

A. Pneumonia in the left lower lobe (blue arrow) four days after the second cisplatin/paclitaxel chemotherapy (Jul. 18, 2020). B. Nearly complete resolution of the pulmonary metastasis and pneumonia eight days prior to the fourth course of cisplatin/gemcitabine chemotherapy (Jan. 6, 2021). 


\section{Cureus}

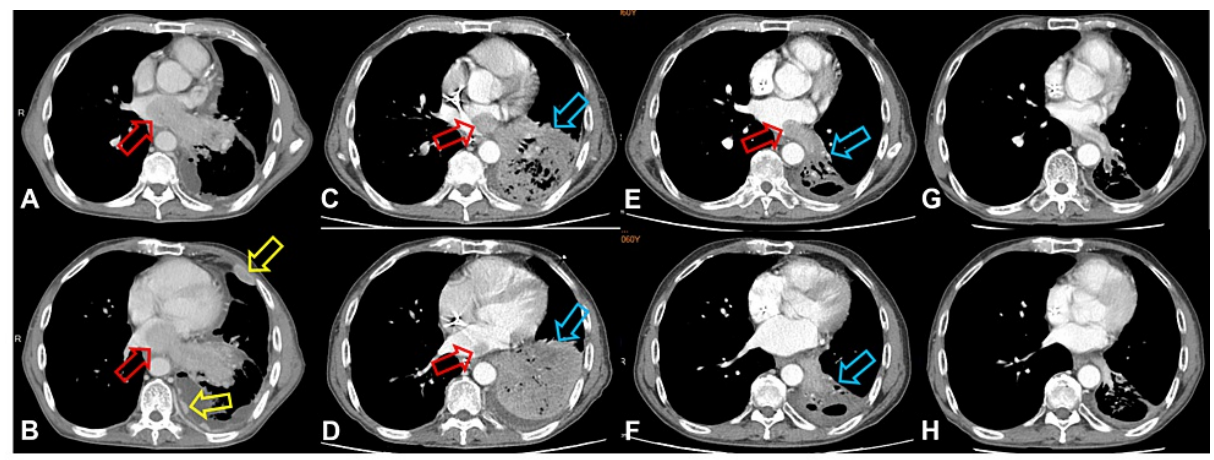

FIGURE 7: CT scan of chest during the whole treatment course.

A and B. At initial presentation (May 11, 2020). C and D. Follow-up study after two courses of cisplatin/paclitaxel chemotherapy (Jul. 22, 2020). E and F. Follow-up study after four courses of cisplatin/paclitaxel chemotherapy (Oct. 7, 2020). G and H. Follow-up study after four additional courses of cisplatin/gemcitabine chemotherapy (Feb. 3, 2021). Red arrow: Tumor invasion into the left atrium from left inferior pulmonary veins. Yellow arrow: Pleural metastasis. Blue arrow: Left lower lobe metastatic tumor mixed with pneumonia consolidation.

\section{Discussion}

Traditionally, nasopharyngeal cancer is closely linked to Epstein-Barr virus infection [9] and the only subtype of lung cancer strongly associated with Epstein-Barr virus is lymphoepithelioma-like carcinoma of the lung in Asian patients [10], while our patient's pleural lesions did not belong to this specific lung cancer category. Despite detection of Epstein-Barr virus genome or transcripts in a very small number of lung cancer tissues was reported recently, the incidence, 7 out of 1127, was quite low [11]. We thus tend to believe that late pulmonary and pleural metastases from a preceding nasopharyngeal carcinoma would be a more coherent diagnosis based on the patient's past history and the rarity of Epstein-Barr virus in common lung cancer types.

The incidence of pulmonary metastasis from nasopharyngeal carcinoma is estimated to be 6.8\% [12], and lung metastasis alone has a more favorable prognosis than metastasis to multiple or other sole organs [13]. Nonetheless, an awesome pulmonary metastasis with left atrial extension like our patient's surely would not be considered to have an optimistic prognosis. Fortunately, the lesions responded to cisplatin-based systemic chemotherapy rather well and the risky cardiopulmonary distress was satisfactorily relieved.

Treatment with cisplatin plus paclitaxel as the first-line regimen for metastatic nasopharyngeal carcinoma led to comparable progression-free survival and overall survival to those of other four commonly adopted protocols including cisplatin + fluorouracil, cisplatin + gemcitabine, cisplatin + paclitaxel + fluorouracil, and cisplatin + fluorouracil + bleomycin according to a retrospective analysis [14]. Therefore, for nasopharyngeal carcinoma patients who had already received cisplatin plus fluorouracil as the front-line therapy, we like to recommend cisplatin in combination with either paclitaxel or gemcitabine as the salvage treatment upon metastatic relapse.

Modern induction chemotherapy has been shown to bring left atrial extension from lung cancer into remission successfully and make complete resection of residual pulmonary tumor possible without the aid of cardiopulmonary bypass, reducing the surgical risk to a great degree [15]. It seems confident to say that shrinkage of tumors invading left atrium with intensive systemic chemotherapy should also be the first step of treatment prior to surgical procedures of curative intent for chemotherapy-sensitive metastasis from other primary sites including nasopharyngeal carcinoma. Furthermore, in the era of molecular targeted therapy and immunotherapy, in addition to immune checkpoint inhibitors [16], rapid progress could be expected in the field of developing specific therapeutic agents targeting Epstein-Barr virus-related molecules [17-18], probably rendering invasive resection of even less priority in treating metastatic nasopharyngeal carcinoma a few years from now.

\section{Conclusions}

A malignant tumor could relapse as a distant metastasis long after the primary site was cleared up. Modern pathologic investigation techniques provide tremendous help in identification of the tumor's nature and aid in choosing the best therapeutic modality. Cancer invasion into left atrium along pulmonary veins might respond to adequate systemic therapy very well, making the patient exempt from high risk surgical excision. This case report gives an extremely encouraging demonstration.

\section{Additional Information}




\section{Disclosures}

Human subjects: Consent was obtained or waived by all participants in this study. Ethics Committee Ministry of Health and Welfare Taitung Hospital issued approval Not applicable. No ethical issues are concerned. Written informed consent was obtained from the patient's younger sister for publication of this case report and any accompanying images. . Conflicts of interest: In compliance with the ICMJE uniform disclosure form, all authors declare the following: Payment/services info: All authors have declared that no financial support was received from any organization for the submitted work. Financial relationships: All authors have declared that they have no financial relationships at present or within the previous three years with any organizations that might have an interest in the submitted work. Other relationships: All authors have declared that there are no other relationships or activities that could appear to have influenced the submitted work.

\section{References}

1. Takahashi K, Furuse M, Hanaoka H, et al.: Pulmonary vein and left atrial invasion by lung cancer: assessment by breath-hold gadolinium-enhanced three-dimensional MR angiography. J Comput Assist Tomogr. 2000, 24:557-561. 10.1097/00004728-200007000-00008

2. Ratto GB, Costa R, Vassallo G, Alloisio A, Maineri P, Bruzzi P: Twelve-year experience with left atrial resection in the treatment of non-small cell lung cancer. Ann Thorac Surg. 2004, 78:234-237. 10.1016/j.athoracsur.2004.01.023

3. Funakoshi Y, Mukohara T, Kataoka T, et al.: Left atrial extension of metastatic lung tumor via pulmonary vein: report on the first case of Ewing sarcoma. Rare Tumors. 2010, 2:e53. 10.4081/rt.2010.e53

4. Cochennec F, Seguin A, Riquet M, Fabiani JN: Intracardiac renal cell carcinoma metastasis. Eur J Cardiothorac Surg. 2008, 34:697-699. 10.1016/j.ejcts.2008.05.040

5. Zissin R, Shapiro-Feinberg M, Rachmani R, Kots E: Lung metastasis invading the left atrium--CT diagnosis . Br J Radiol. 1999, 72:1211-1212. 10.1259/bjr.72.864.10703480

6. Reisenauer JS, Said SM, Allen MS: Metastatic rectal adenocarcinoma presenting as a giant left atrial mass: resection combined with right lower lobectomy. Ann Thorac Surg. 2016, 102:e19-e21 . 10.1016/j.athoracsur.2015.11.064

7. Rodriguez A, Roubertie F, Thumerel M, Jougon J: Pulmonary metastatic chondrosarcoma with massive extension into left atrium and left ventricle: outcome of surgical management in emergency. Eur J Cardiothorac Surg. 2013, 44:e341-e342. 10.1093/ejcts/ezt426

8. Lee S, Park IK, Cho SH: Extension of metastatic lung cancer from cervix cancer through pulmonary vein into the left atrium. Ann Thorac Surg. 2011, 92:1131. 10.1016/j.athoracsur.2011.03.057

9. Tsao SW, Tsang CM, Lo KW: Epstein-Barr virus infection and nasopharyngeal carcinoma. Philos Trans R Soc Lond B Biol Sci. 2017, 372:20160270. 10.1098/rstb.2016.0270

10. Hu Y, Ren S, Liu Y, Han W, Liu W: Pulmonary lymphoepithelioma-like carcinoma: a mini-review . Onco Targets Ther. 2020, 13:3921-3929. 10.2147/OTT.S241337

11. Kheir F, Zhao M, Strong MJ, et al.: Detection of Epstein-Barr virus infection in non-small cell lung cancer . Cancers (Basel). 2019, 11:759. 10.3390/cancers11060759

12. Zhao W, Lei H, Zhu X, Li L, Qu S, Liang X: Investigation of long-term survival outcomes and failure patterns of patients with nasopharyngeal carcinoma receiving intensity-modulated radiotherapy: a retrospective analysis. Oncotarget. 2016, 7:86914-86925. 10.18632/oncotarget.13564

13. Hui EP, Leung SF, Au JS, et al.: Lung metastasis alone in nasopharyngeal carcinoma: a relatively favorable prognostic group. A study by the Hong Kong Nasopharyngeal Carcinoma Study Group. Cancer. 2004, 101:300-306. 10.1002/cncr.20358

14. Jin Y, Shi YX, Cai XY, et al.: Comparison of five cisplatin-based regimens frequently used as the first-line protocols in metastatic nasopharyngeal carcinoma. J Cancer Res Clin Oncol. 2012, 138:1717-1725. 10.1007/s00432-012-1219-x

15. Toyooka S, Mori H, Kiura K, Date H: Induction chemoradiotherapy prior to surgery for non-small cell lung cancer invading the left atrium. Eur J Cardiothorac Surg. 2008, 33:315-316. 10.1016/j.ejcts.2007.10.022

16. Johnson D, Ma BBY: Targeting the PD-1/ PD-L1 interaction in nasopharyngeal carcinoma . Oral Oncol. 2021, 113:105127. 10.1016/j.oraloncology.2020.105127

17. Hau PM, Lung HL, Wu M, et al.: Targeting Epstein-Barr virus in nasopharyngeal carcinoma . Front Oncol. 2020, 10:600. 10.3389/fonc.2020.00600

18. Huang J, Fogg M, Wirth LJ, et al.: Epstein-Barr virus-specific adoptive immunotherapy for recurrent, metastatic nasopharyngeal carcinoma. Cancer. 2017, 123:2642-2650. 10.1002/cncr.30541 